\title{
Accumulated Reconstruction Method for Electron Holography
}

\author{
Ken Harada ${ }^{1}$ and Hiroto Kasai ${ }^{1}$ \\ ${ }^{1}$ Central Research Laboratory, Hitachi Ltd., Hatoyama, Saitama 350-0395, JAPAN
}

One of the long standing problems affecting electron holography, such as lateral coherence limitation, has been solved by the split illumination method with a specially customized transmission electron microscope (TEM). The customized TEM has single [1] or double [2] biprisms in a condenser optical system. Conventional TEM, constructed for electron holography, however, does not have any biprisms in the condenser system. The problem, therefore, still remains unsolved in practice. In order to reduce the difficulties of conventional electron holography, an "accumulated reconstruction" method depending on the principle of two-wave interferometry was developed.

Figure 1 explains the idea. The target sample is in the object region (n) far from the specimen edge, and the distance from the region (n) to the vacuum region (ref) exceeds the coherent length R". The first hologram is recorded with two waves in region (n) and region (n-1); the second hologram is also recorded with these waves in regions (n-1) and (n-2). The specimen or biprism is shifted perpendicular to the hologram through a distance $W$ that is equal to the hologram's width. The last hologram is recorded with the waves in region (1) and the reference region (ref). After reconstruction of all of the holograms, the reconstructed phase distributions $\Delta \eta_{\mathrm{n}}=\eta_{\mathrm{n}}-\eta_{\mathrm{n}-1}$ are summed one by one, as follows:

$$
\sum \Delta \eta_{n}=\sum\left(\eta_{n}-\eta_{n-1}\right)=\eta_{n}-\eta_{\text {ref }}=\eta_{n}
$$

where $\eta_{\mathrm{n}}$ is the phase distribution of each wave. Equation 1 means that the summed result is the same as the phase distribution reconstructed from the hologram recorded in region (n) and the reference region (ref). The problem is thus solved in principle. By use of the accumulation method, the other regions are reconstructed as the hologram recorded using the reference region (ref). The method has another advantage; i.e., all the regions from (n) to (1) are reconstructed by using just one reference wave. Wide area reconstruction under standard conditions can thus be realized.

Figure 2 shows a conventional phase map by double biprism interferometry [3], corresponding to the magnetic lines of force from the apex of an MFM tip. When the hologram width was changed, the distribution of the magnetic lines of force changed. This clearly means that the magnetic field from the tip leaked into the reference region. Thus keeping the reference wave far from the magnetic material is very important.

Figure 3 shows a multi-exposure micrograph that has been subject to image processing. In each exposure, the tip was shifted a regular distance equal to the hologram width $W$. The interference region shown as the white band in Fig. 3 was fixed in each micrograph.

Figure 4 shows the phase distribution reconstructed by the accumulation method for wide area observation. The reconstructed area which was put in order was seven times as wide as the possible area reconstructed by conventional holography. Accordingly, magnetic lines of force from not only the tip 
apex, but also other areas can be visualized. A comparison of the results in Fig. 4 and Fig. 2 indicates that the conventional reconstruction expressed the differentiation of the magnetic field.

\section{References:}

[1] T. Tanigaki et al., Appl. Phys. Lett., 101, 043101 (2012).

[2] T. Tanigaki et al., Ultramicroscopy, 137, 7 (2014).

[3] K. Harada et al., Appl. Phys. Lett., 84, 3229 (2004).

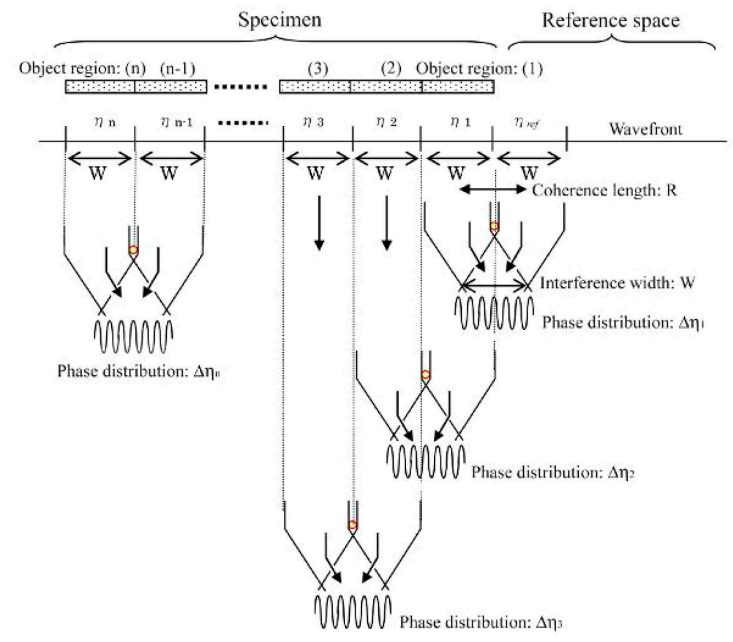

Figure 1.

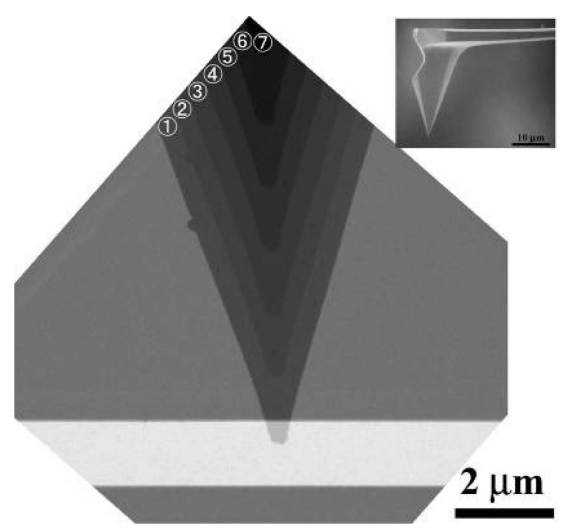

Figure 3.

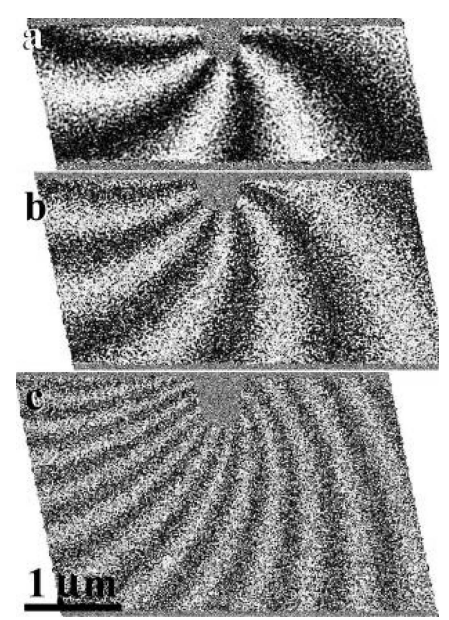

Figure 2.

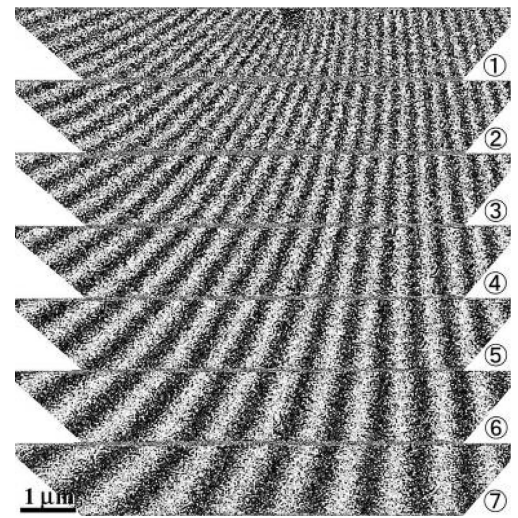

Figure 4.

Figure 1. Principle of "accumulated reconstruction".

Figure 2. Magnetic lines of force $(\times 4)$ from MFM tip from conventional reconstruction.

Figure 3. Multi-exposure micrograph. The tip was shifted at regular intervals equal to the hologram width.

Figure 4. Magnetic lines of force $(\times 1)$ around the tip reconstructed by the accumulation method. 